\title{
Noninterventional Therapies for the Management of Knee Osteoarthritis
}

\author{
${ }^{1}$ Department of Physical Medicine and Rehabilitation, McGaw \\ Medical Center of Northwestern University, Chicago, Illinois \\ ${ }^{2}$ Department of Physical Medicine and Rehabilitation, Shirley Ryan \\ Ability Lab, Chicago, Illinois \\ ${ }^{3}$ Northwestern University Feinberg School of Medicine, Chicago, Illinois \\ ${ }^{4}$ Department of Physical Medicine and Rehabilitation, Northwestern \\ University Feinberg School of Medicine, Chicago, Illinois
}

Joseph Dadabo, MD ${ }^{1,2}$ Julia Fram, BA ${ }^{3}$ Prakash Jayabalan, MD, PhD ${ }^{2,4}$

Address for correspondence Prakash Jayabalan, MD, PhD, Department of Physical Medicine and Rehabilitation, Shirley Ryan Ability Lab, 355 East Erie Street, Chicago, IL 60611 (e-mail: pjayabalan@sralab.org).

J Knee Surg 2019;32:46-54.

Abstract
Keywords
- knee osteoarthritis
- exercise
- modalities
- pain

The goal of the practitioner managing a patient with knee osteoarthritis (OA) is to minimize pain and optimize their function. Several noninterventional (noninjectable) therapies are available for these individuals, each having varying levels of efficacy. An individualized approach to the patient is most beneficial in individuals with knee $\mathrm{OA}$ and the treatment plan the practitioner chooses should be based on this principle. The focus of this article is to provide an up-to-date overview of the treatment strategies available, evidence to support them, and in whom these treatments would be most appropriate. These include exercise (aerobic and resistance), weight loss, bracing and orthotics, topical and oral analgesic medications, therapeutic modalities, and oral supplements.
Osteoarthritis (OA) of the knee is a multifaceted challenge for physicians practicing in both primary care and specialty settings. This condition causes pain and secondary functional decline as the disease progresses over time. In the United States, symptomatic knee OA has been reported in $10 \%$ of men and $13 \%$ of women over the age of $60 .^{1}$ Radiographic evidence of OA shows an even higher prevalence, suggesting that the population at risk is broader. ${ }^{2,3}$ Indeed, knee and hip OA represent the most common cause of walking-related disability in the United States. ${ }^{4}$ Surgical management is typically reserved for those with end-stage disease and currently total knee arthroplasty is the most commonly performed inpatient surgical procedure in the United States. ${ }^{5}$ For those with mildto-moderate disease, prevailing treatment options have been grouped broadly under the umbrella of "non-operative" management. Within this category there exists a myriad of treatment options, among them exercise protocols, weight loss, dietary supplements, topical and oral medications, corticosteroid, and biologic injections. ${ }^{6}$ In this review, we will outline evidence-based noninterventional (noninjection) treatment

received

June 6, 2018

accepted after revision

October 15, 2018

published online

November 26, 2018 strategies for the management of knee OA. In this review, we highlight the most effective approaches and clarify common misconceptions as they relate to both disease symptomatic progression and appropriate management principles focused on pain reduction and preservation of function.

\section{Exercise Prescription}

Physical activity has proven to have benefit for both pain and physical function limitations. ${ }^{7}$ In fact, exercise more generally has demonstrated hypoalgesic effects across a range of chronic musculoskeletal pain conditions, including fibromyalgia, nonspecific low back pain, and OA of various joints. ${ }^{8-10}$ However, there are currently no clearly defined parameters for the nature, frequency, duration, and intensity of physical activity treatment programs in patients with knee OA. Furthermore, there is an important distinction between supervised activity with a physical therapist and independent home exercise programs directed by patients without the presence of trained clinical providers. ${ }^{11}$

Copyright @ 2019 by Thieme Medical Publishers, Inc., 333 Seventh Avenue, New York, NY 10001, USA. Tel: +1(212) 584-4662.
DOI https://doi.org/ $10.1055 / \mathrm{s}-0038-1676107$. ISSN $1538-8506$. 
Broadly, the aim of physical activity interventions in patients with knee $\mathrm{OA}$ is pain reduction and preservation of function. Within this framework, there exists more specific goals of quadriceps muscle strengthening, joint stabilization, maintaining range of motion, and improving aerobic exercise capacity. ${ }^{12,13}$ Patients and providers alike are sometimes apprehensive about the potential for exercise to precipitate further harm to the joints. However, there is limited evidence to suggest that physical activity causes or exacerbates symptomatic knee OA. In fact, recent reviews have demonstrated largely positive effects from such activity interventions. ${ }^{7,14}$ The implementation of formalized home exercise programs has been shown to reduce pain immediately following treatment, with sustained improvement over a period of weeks to months. Relatedly, exercise in this population is also tied to improvements in physical function and self-reported quality of life. $^{15}$

Physical activity is a general term, denoting voluntary bodily movement that requires the expenditure of energy. ${ }^{16}$ There are several formal and informal activities that might be described as exercise; however, a narrow range of activities has evidence supporting their use in the treatment of OA. Activities with the strongest evidence in treating patients with knee OA include aerobic exercise and resistance strength training. ${ }^{14,15}$

\section{Aerobic Exercise}

Aerobic exercise is characterized by sustained physical activity that requires oxidative metabolism for energy generation. ${ }^{17}$ Swimming, cycling, and jogging all fall within this category. Walking is the most accessible form of aerobic exercise, and there is strong evidence to support its use for reducing pain and improving physical function in patients with knee OA. ${ }^{18}$ Formal walking programs have been shown to improve walking performance, increase quadriceps muscle strength, and reduce OA-associated knee pain. ${ }^{19,20}$ Across various trials, treatment typically includes 20 to 60 minutes of continuous walking two to three times per week, often supplemented with light stretching activities, body-weight support training, and patient education on related health topics. Pain reduction immediately following treatment is not uncommon, although the effect can be small and improvement sometimes requires months to observe. ${ }^{15}$ Furthermore, the benefits of walking exercise tend to diminish following cessation of physical activity, underscoring the importance of sustained adherence for treatment success. ${ }^{21,22}$ Serious adverse events are rare, although some patients do experience worsening of joint pain following activity. ${ }^{15,23}$

In addition to walking, other higher intensity aerobic activities such as running and cycling have also been investigated for possible benefit with regard to pain and functional impairment. A recent systematic review highlighted a dearth of evidence related to exercise intensity and found low- to very low-quality evidence for clinical benefit of high intensity relative to low intensity exercise in patients with knee OA. ${ }^{24}$ However, while exercise intensity level may not be strongly tied to pain reduction or functional improvement, there is relatively strong evidence supporting alternative forms of aerobic exercise for patients with knee OA. For example, recent evidence has suggested the clinical benefit of aquatic aerobics. The buoyancy of water reduces weight-bearing stress on the joints and may contribute to a greater degree of pain reduction relative to landbased aerobics. ${ }^{25}$ The aquatic environment also provides light resistance for muscle strengthening and, in heated pools, potentially therapeutic relief of associated soft tissue pain and muscle spasms. ${ }^{26}$ Multiple randomized controlled trials have demonstrated analgesic effects following implementation of formal aquatic exercise programs. ${ }^{26-28}$ The specific routines varied slightly from study to study, but as with the walking programs described previously, typically consisted of supervised activity in a heated pool two to three times weekly for 30 to 60 minutes at a time over a period of 6 to 8 weeks. ${ }^{29}$ Altogether, these findings suggest that aerobic exercise, both land and aquatic-based, is safe and effective for knee OA patients there are still a lack of comprehensive guidelines.

\section{Resistance Training}

Resistance training stands alongside aerobic exercise as having the strongest supporting evidence in the treatment of knee OA. ${ }^{30}$ Individuals with knee OA are known to have relative weakness across multiple muscle groups, including the quadriceps, hamstrings, and hip abductors. Importantly, this weakness is associated with functional impairment. ${ }^{31}$ Resistance strength exercises can target these individualized muscle groups, while also providing a measure of global endurance training in much the same manner as a walking program. Typical routines are comprised of isotonic knee extension and flexion exercises with ankle weights for resistance, body-weight squats and step-ups, and isometric quadriceps contraction in a supine position with the knees held in extension. Often, flexibility, stretching, and dynamic balance activities were incorporated into the routine and as with some of the aerobic regimens outlined above, frequency was around two to three times per week over a period of 6 to 12 weeks. $^{30,32,33}$ Importantly, the benefits of such strengthening, flexibility, and balance activities have been shown to improve functional outcomes even in those patients who ultimately undergo total knee arthroplasty, suggesting clinical benefit across a broad range of disease severity. ${ }^{34,35}$

Patients sometimes adopt a multimodal approach to physical activity, participating in several different types of exercise, while others restrict their routine to a single activity. In guiding patients on the implementation of an exercise program, the focus should be on finding evidencebased activities that they are able to perform on a sustained basis. Rather than ordering a narrowly defined set of exercises, the provider should work together with the patient to identify prior exercise experiences, preferences, underlying motivations, and community resources so as to craft an exercise prescription that is individualized and precise. This collaborative approach is more likely to be adopted by the patients, many of whom may not otherwise be inclined toward physical activity due to chronic pain and disability from their disease. Moreover, it is also in keeping with a patient-centered approach to management that is more likely to be adhered to by the patient (-Fig. 1). 


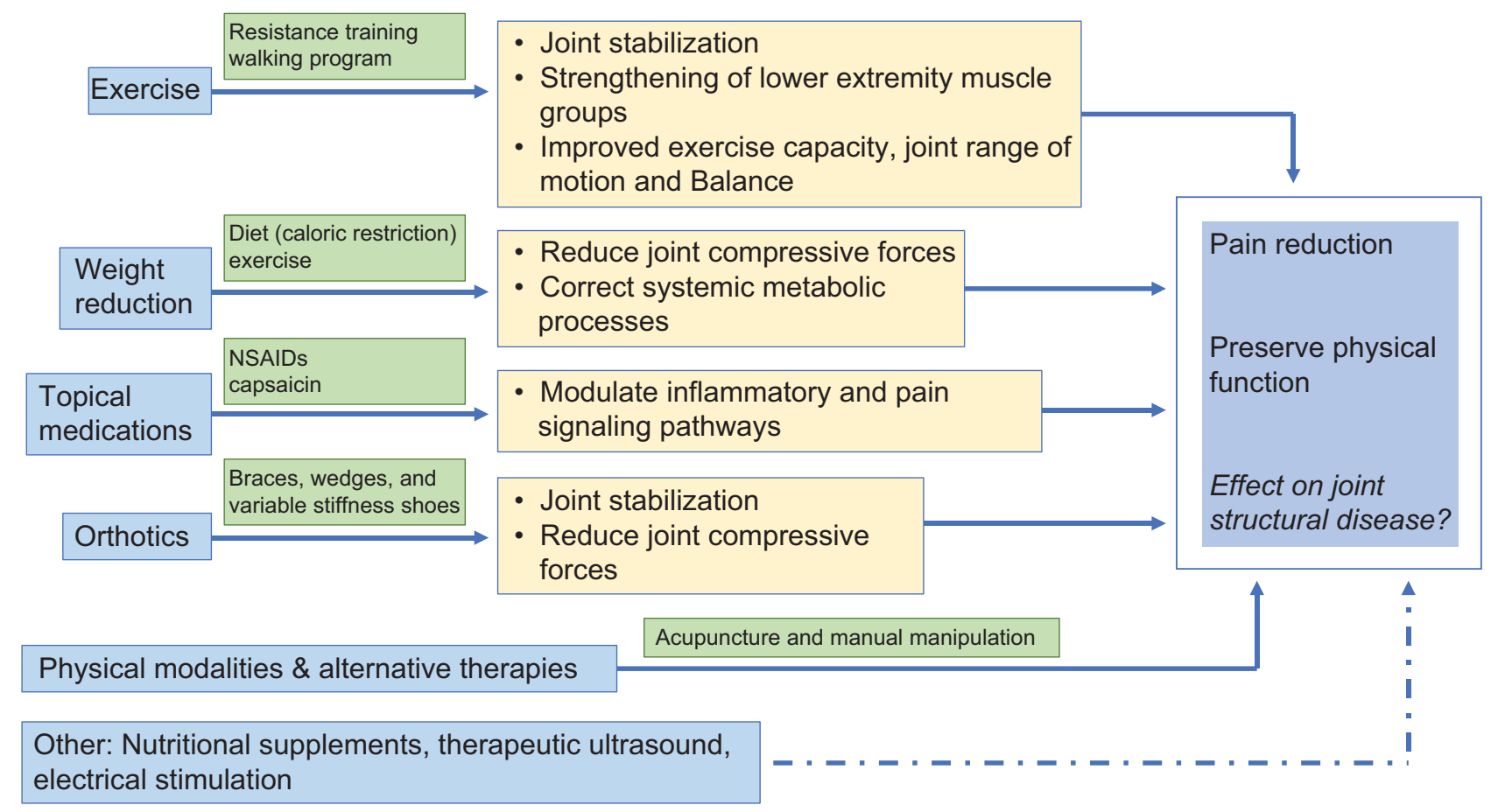

Fig. 1 Summary diagram of non-interventional therapies for knee osteoarthritis featured in this review.

\section{Weight Loss}

Overweight status and obesity, defined as a body mass index (BMI) of 25 to $29.9 \mathrm{~kg} / \mathrm{m}^{2}$ or $\geq 30 \mathrm{~kg} / \mathrm{m}^{2}$ respectively, are prominent risk factors for the development and progression of knee OA as well as the need for joint replacement. ${ }^{36,37}$ Weight reduction is a fundamental component of nonsurgical management of knee OA in individuals with excess weight. A meta-analysis found that a 5 unit increase in BMI translates to a $35 \%$ increased risk of knee $\mathrm{OA}^{38}$ The pathophysiology underlying this association is thought to be product of mechanical and metabolic factors, with excess weight causing increased joint loading, relative muscle weakness, and adiposity-related systemic inflammatory proteins, known as adipokines. ${ }^{39}$

Weight loss as a treatment strategy is supported by several randomized control trials demonstrating that lifestyle modifications, primarily changes in diet and exercise, are safe and effective therapies in this population, yielding clinically significant improvements in pain and physical function. ${ }^{40-43}$ The Intensive Diet and Exercise for Arthritis trial investigated the effect of 18 months of a calorie restriction and partial mealreplacement-based diet or exercise, or a combination of both, in overweight individuals with knee OA $(n=419)$. This study showed that the loss of over $10 \%$ of baseline body weight resulted in significant increases in self-reported pain, function, and mobility as measured by the 6 -minute walk test and gait speed. Additionally, researchers found decreased knee compressive forces and serum concentrations of interleukin-6, an inflammatory mediator) among individuals who lost $>10 \%$ of body weight. This lends support to the potential roles of weight loss in modulating mechanical and inflammatory pathways underlying knee OA. ${ }^{44,45}$
The effects of weight loss on joint structure and progression of established disease are undetermined. A recent study found that following bariatric surgery for weight loss in 40 obese individuals (who lost $>20 \%$ body weight post-surgery), there were no significant structural joint changes on magnetic resonance imaging (MRI), although there were noted improvements in pain and function. ${ }^{46}$ Alternatively, Gersing et al found a reduction in cartilage damage on MRI among overweight and obese persons $(n=640)$ with risk factors of or mild radiographic findings of knee OA who lost at least $10 \%$ of their body weight over 4 years compared with those who remained at a stable weight. ${ }^{47}$ Both of these studies focus on persons with risk factors or early signs of knee OA. In individuals with established radiographic knee OA studies, a 16-week low energy diet program $(n=192)$ with the goal of $>10 \%$ weight loss found significant symptomatic improvement, in those who achieved this target. ${ }^{48}$

When counseling a patient with comorbid excess weight and knee OA on weight reduction, it is crucial to agree on an individualized weight loss goal and strategy. The National Heart, Lungs, and Bones Institute recommend that overweight and obese adults attempt to lose $10 \%$ of baseline weight within 6 months at the beginning of a weight loss program. ${ }^{49}$ In individuals with knee OA, a meta-analysis demonstrated that weight reduction of $5.1 \%$ of body weight at the rate of $0.24 \%$ per week significantly improved disability in overweight and obese patients. ${ }^{42}$ While increased weight reduction correlates with more significant symptomatic improvement, given the degree of difficulty of weight loss, it is important to note that patients are likely to experience improvements in pain and disability with any degree of weight loss. 
Initial weight reduction strategy should consist of lifestyle interventions with the ultimate aim of establishing a negative energy balance. Evidence supports the utility of caloric restriction for weight loss in knee OA. While opinions on the optimal diet composition are varied and lacking in conclusive evidence, the combination of a calorie-restricted diet and exercise is the optimal approach to weight reduction with the aim of addressing patient pain and disability. Of note, in several trials assessing the role of weight loss on joint-related symptoms, improvements in pain and function were greatest when a combined weight loss strategy of diet and exercise was implemented rather than either alone. ${ }^{44,48,50}$ A patient-centered approach to weight reduction represents a promising intervention to reduce pain, improve physical function, and potentially impact the progression of knee OA among persons with excess weight.

\section{Orthotics}

Knee orthoses represent an important category for consideration in noninterventional management of OA. The diversity of available options reflects the many varied approaches toward joint stabilization and joint stress reduction. Whereas formalized walking programs and resistance strength training have a fairly robust body of randomized controlled trials supporting their use, the evidence regarding knee orthoses is of varying quality. $^{51}$

\section{Knee Braces}

Knee braces are characterized by a diverse set of orthoses with the intent of offloading joint stress and stabilizing knee alignment. A systematic review from 2011 found there was moderate quality evidence demonstrating that knee braces of all types were associated with reductions in pain and joint stiffness as well as improved walking mechanics. ${ }^{52}$ However, a subsequent review in 2015 identified a general lack of evidence related to the efficacy of such devices. ${ }^{53}$ Under fluoroscopic surveillance, properly fitted braces have been shown to increase articular separation, consistent with the notion that these devices offload degenerative compartments in the knee. ${ }^{54}$ Such braces have also been shown to improve proprioception, although the degree of difference and clinical significance is questionable. ${ }^{55}$ Nonetheless, there is evidence that the global reduction in pain associated with consistent use of knee braces may facilitate correction of gait asymmetry and improvement in function. ${ }^{56}$ Although promising data exist, the variability across these different trials suggests that more robust clinical trials are needed before an unequivocal recommendation can be made on behalf of knee braces for the treatment of $\mathrm{OA}$.

\section{Wedge Insoles, Orthotic, and Walking Shoes}

Relatedly, wedge insoles and specialized orthotic walking shoes also have a mixed body of supporting evidence. In patients with medial compartment OA, lateral wedge insoles worn consistently for 12 months did not lead to reduction in pain or structural differences in tibial cartilage volume. ${ }^{57}$ Similarly, a trial examining patients using wedge insoles for 2 years failed to show any difference in pain or disability over that period. However, participants in the treatment group of this latter study tended to utilize nonsteroidal anti-inflammatory drugs (NSAIDs) less frequently than those in the control arm. ${ }^{58} \mathrm{~A}$ systematic review from 2012 not only acknowledges the relative lack of favorable evidence for lateral wedge insoles but also suggests that they may be of particular benefit in a subset of patients with higher BMI and earlier stages of disease. ${ }^{59}$ This distinction is important, as obese patients are often poorly suited for knee braces or other compression sleeves because of an inability to achieve proper fit. ${ }^{54}$

Variable stiffness walking shoes represent another alternative to traditional knee braces or wedge insoles. A trial that followed patients for 6 months found that consistent use of variable stiffness shoes led to reduction in pain and adduction moment at the knee as well as improvement in physical function. ${ }^{60} \mathrm{~A}$ follow-up study by the same authors demonstrated continued reduction in adduction moment after 12 months, although there were no longer differences between the treatment and control groups with regard to pain or disability. ${ }^{61}$ A larger systematic review similarly found that knee adduction moment was reduced with the use of variable stiffness shoes. ${ }^{62}$ In medial compartment disease, reducing stress on that component of the joint is a potentially beneficial biomechanical alteration. However, at this time further evidence is needed to show that such changes in stance and gait ultimately bear a concordant response in pain and physical function. At present, as is the case with braces and wedge insoles, a lack of confirmatory data prevents a full recommendation on behalf of variable stiffness shoes.

\section{Therapeutic Modalities}

In addition to therapeutic exercise programs and weight loss, there are several complementary and alternative interventions that some have used to alleviate pain and disability associated with knee OA. Although not exhaustive, this list includes such diverse modalities as acupuncture, ultrasound, electrical stimulation, and manual manipulation. There are varying degrees of support for each of these respective treatments, but in all cases the literature would benefit from a larger number of high-quality randomized, doubleblinded, placebo-controlled trials. The section that follows will touch briefly on each of the options noted above, detailing the weight of current evidence and highlighting pertinent precautions and contraindications.

\section{Acupuncture}

There have actually been numerous comparative trials evaluating the efficacy of acupuncture for reducing pain and improving physical function for knee OA. Some of these trials have utilized sham procedures as an experimental control, while others have set acupuncture against alternative treatment programs or no intervention. Both types of trials have shown positive results, although the effect size tended to be larger in the latter group (no intervention). ${ }^{51}$ Interpretation of these trials is limited, however, as there was a potential placebo effect at work in the treatment arm of those trials that offered no 
alternative intervention. Relatedly, in the case of sham acupuncture trials, the effect size was typically small and may not actually represent a clinically significant difference. ${ }^{63}$ The lack of clear anatomical and physiological correlates for needle placement and the varying techniques employed by practitioners across different geographic locations have potentially impacted the outcome of clinical trials. ${ }^{64,65}$

\section{Therapeutic Ultrasound}

Therapeutic ultrasound is often applied in conjunction with traditional physical therapy programs targeting knee stability, quadriceps strengthening, and general cardiovascular fitness. A randomized clinical trial from 2012 comparing ultrasound to a sham procedure found that patients in both the treatment and control groups eventually experienced pain relief and improvements in physical function while enrolled in physical therapy. ${ }^{66}$ However, there was no statistically significant difference between the two groups. This is consistent with two larger systematic reviews, which suggest that ultrasound may be associated with modest benefit but that higher quality evidence is needed before drawing conclusions. ${ }^{67,68}$ There is similarly limited evidence to support neuromuscular electrical stimulation for the treatment of knee OA. A review from 2012 found inconsistent trial data for the practice; however, the American College of Rheumatology (ACR) does make a conditional recommendation for its use in patients with moderateto-severe OA pain who are unable or unwilling to undergo total knee arthroplasty. ${ }^{69,70}$ As with acupuncture, adverse effects are generally rare with the use of therapeutic ultrasound and electrical stimulation. There is some disagreement about the contraindications for use, but in general these procedures should not be used over sites of infection or active bleeding, metal hardware, or implanted devices such as pacemakers. ${ }^{71,72}$

Manual manipulation by a trained physical therapist is also sometimes utilized as an adjuvant treatment alongside other forms of physical therapy and exercise training programs in the treatment of knee OA. Such interventions typically involve passive knee range of motion and joint mobilization. A systematic review of 12 randomized controlled trials found there to be moderate effect on pain reduction with manual manipulation and exercise together relative to smaller effects associated with exercise therapy or strength training alone. ${ }^{73}$ There was heterogeneity across the trials included in this review, however, with some patients receiving therapeutic ultrasound, formal education, or group therapies in addition to routine strengthening activities. $^{74,75}$ In assessing the current literature, there appears to be modest benefit and minimal risk associated with manual manipulation. ${ }^{51}$ Still, higher quality evidence would be helpful in developing more cogent guidelines for its clinical application in conjunction with other forms of passive and active physical training.

\section{Topical and Oral Analgesic Medications}

Alongside physical activity, weight loss, and bracing, topical and oral medications are sometimes indicated in treating patients with knee OA. The goals of pharmacological inter- vention are similar to those of the other modalities listed, namely, pain reduction and preservation of function. As with any potential treatment, the provider must weigh the likelihood of clinical benefit against the risk of adverse effects. Unlike exercise or bracing, medications are uniquely situated in that they bear potential for rapid onset of effect but also systemic organ toxicity. Additionally, in the case of opiates and certain neuropathic pain agents, there is further risk of drug tolerance, dependence, and withdrawal. This section will outline a comprehensive approach to medication management in patients with knee $\mathrm{OA}$, highlighting evidence-based treatment principles as well as common prescription pitfalls.

Many of the drug classes commonly used to treat knee OA and general chronic musculoskeletal pain can be associated with vital organ toxicity. ${ }^{76}$ This is true even in the absence of overdose or drug misuse. ${ }^{77}$ Minimizing drug toxicity should be a priority for any provider treating patients with chronic pain and functional impairment. Topical medications are advantageous in this regard, as they carry a much lower risk of systemic absorption than oral agents while also providing targeted relief to painful joints. ${ }^{78}$ For this reason, they are often a useful starting point for patients who have pain refractory to other conservative measures such as bracing or icing. Topicals can sometimes be used as analgesic-sparing agents, but in other situations may be prescribed in combination with oral medications. ${ }^{79,80}$ Diclofenac gel is perhaps the best studied and most strongly supported topical therapy for musculoskeletal joint pain. Several randomized clinical trials have demonstrated efficacy of topical diclofenac in reducing pain relative to placebo. ${ }^{81,82}$ Relatedly, ketoprofen has also been shown to be helpful at alleviating pain in this population. ${ }^{83}$ Both agents have received conditional recommendation by the ACR for the treatment of knee OA. ${ }^{70}$ Topical NSAIDs are typically better tolerated than their oral counterparts. While there is risk of localized skin irritation, xerosis, and pruritus, systemic absorption is minimal in comparison to oral agents and therefore there is far less risk of gastrointestinal upset, renal dysfunction, and cardiotoxicity as is often associated with long-term use of oral NSAIDs. ${ }^{80,82,83}$

Whereas NSAIDs are known to inhibit the enzyme cyclooxygenase, capsaicin is thought to deplete neuronal stores of substance $P$, thereby reducing transmission of nociceptive pain signals to the central nervous system. Several placebo-controlled trials have shown relative benefit with the use of topical capsaicin, although uncomfortable burning at the site of application was a commonly reported side effect. ${ }^{84,85}$ Topical lidocaine has demonstrated some benefit, although a robust body of evidence is lacking. ${ }^{86}$ Camphor, menthol, and salicylates are also sometimes utilized, although evidence for or against these latter agents is similarly limited. ${ }^{79}$

If pain relief is not sufficient with topical therapies alone, the next consideration is oral medication. Acetaminophen is more effective than placebo at alleviating pain from OA and carries lower risk of systemic toxicity than oral NSAIDs, although liver failure may result from doses greater than 3 to $4 \mathrm{~g}$ per day. ${ }^{87}$ In patients that do not respond to acetaminophen, oral NSAIDs are an appropriate alternative. Both nonselective agents such as ibuprofen or naproxen as well as 
cyclooxygenase-2 (COX-2)-selective drugs such as celecoxib have been shown to reduce pain in patients with knee OA. ${ }^{70,87}$ However, these medications should not be administered to patients with a history of chronic kidney disease because there is a further risk of renal impairment. A recent history of gastrointestinal bleed or gastric ulceration is a relative contraindication, although COX-2 inhibitors such as celecoxib may still be appropriate for these patients. ${ }^{70,87,88}$ Advanced age is another reason for caution and in fact, the ACR advises against use of oral NSAIDs in patients $>75$ years old. ${ }^{9}$ These various precautions reflect the potential complications that may arise from systemic administration of NSAIDs. Such effects are even more likely with long-term use, and it is important to constantly assess the necessity of oral NSAIDs and determine on an ongoing basis whether alternative treatment options may be similarly effective. ${ }^{87,88}$

In recent years, tricyclic antidepressants (TCAs) and serotonin norepinephrine reuptake inhibitors have been used with greater frequency in patients with chronic musculoskeletal pain. ${ }^{89}$ While these agents are often targeted toward neuropathic pain specifically, they also have underlying usefulness in treating comorbid depression and have been studied in the treatment of osteoarthritic pain. ${ }^{70,89}$ There is some evidence from randomized controlled trials to suggest that duloxetine, a serotonin norepinephrine reuptake inhibitor, may help to reduce pain in patients with knee OA. ${ }^{90}$ Relatedly, use of TCAs such as amitriptyline and nortriptyline for OA pain is currently under study. ${ }^{91,92}$ However, with limited evidence to-date the ACR makes no recommendation for or against the use of duloxetine nor does it comment on the use of TCAs in this population. ${ }^{70}$ Other consensus guidelines have similar recommendations, focusing instead on topicals, acetaminophen, and nonsteroidals. ${ }^{87,88}$

Opioid medications are generally reserved for patients with persistent, severe pain refractory to topical analgesics, acetaminophen, and oral NSAIDs. This relates in part to the potential for drug tolerance and dependence. However, even in patients who respond well to narcotics there are undesirable side effects such as constipation, respiratory depression, and sedation. ${ }^{93}$ Further caution applies in elderly patients and those taking other agents with similar risks, such as benzodiazepines. Tramadol is a weak mu opioid receptor agonist with secondary serotonergic effects that has been studied in the treatment of $O A$ patients. Previous literature shows positive effects with regard to pain reduction, but the effect is generally small. ${ }^{94}$ The ACR makes a conditional recommendation for use of tramadol in knee OA alongside oral NSAIDs and topical pain relievers. The authors were unable to make such advisement for other opioid medications because of limited evidence supporting efficacy, although an exception exists for patients who are unwilling or unable to undergo total knee arthroplasty. ${ }^{70}$ Previous trials have found small, but statistically significant pain reduction in patients taking opioids relative to placebo. However, these same studies also demonstrated heightened risk of adverse effects, including hospitalization and death. ${ }^{95}$ Indeed, the efficacy of chronic opioids for musculoskeletal pain is questionable and the associated risks are relatively high. ${ }^{93,96}$ The potential for undesirable and dangerous side effects renders opioids appro- priate only in a limited number of cases; specifically, in those patients with stringent contraindications to alternative pharmacological options or those with severe, acute pain flares refractory to other conservative measures. Long-term use is not advisable in most cases and in those patients on chronic opioid therapy for knee OA, efforts should be made to slowly wean as tolerated in favor of alternative treatments.

\section{Dietary Supplements}

\section{Glucosamine and Chondroitin Sulfate}

In addition to prescription therapies, there are a variety of nutritional supplements with purported benefit in patients with knee OA. Perhaps the best known of these supplements are glucosamine and chondroitin. The Glucosamine/chondroitin Arthritis Intervention Trial was a randomized controlled trial that compared knee OA patients taking glucosamine and chondroitin alone or in combination with those taking placebo. The study found no significant difference in pain reduction with the use of the nutritional supplements relative to placebo, although the authors noted that glucosamine and chondroitin together may be effective in the subgroup of patients with moderate-to-severe knee pain at baseline. ${ }^{45}$ These findings are consistent with the broader body of literature around glucosamine and chondroitin in patients with $\mathrm{OA}$. While there are some smaller trials demonstrating positive effects in pain reduction, larger analyses have failed to show consistent benefit. ${ }^{97,98}$ The safety profile of both agents is fairly benign and adverse effects associated with their use are generally rare, but the purported benefits have not been consistently supported in the literature. ${ }^{99}$ Indeed, the ACR holds a conditional recommendation that providers should not use glucosamine and chondroitin for the treatment of knee OA. ${ }^{70}$ Other consensus guidelines echo this position, highlighting prior equivocal findings and the relative lack of strong evidence to support their use. ${ }^{51}$

\section{Turmeric}

Turmeric is another dietary supplement thought to have therapeutic effects in OA separate and apart from glucosamine and chondroitin. Curcumin is the primary constituent of turmeric and is often found as an additive to various food items in Southeast Asian cuisine. Curcumin has anti-inflammatory and antioxidative properties that may protect joints from the degenerative processes that characterize OA. ${ }^{100}$ To date, there are a limited number of randomized controlled trials comparing curcumin to placebo in patients with OA. ${ }^{101}$ Those studies are generally small, but they do show modest improvements in pain among patients with degenerative joint disease. The safety profile of turmeric in these trials was favorable and serious side effects were rare. ${ }^{102,103}$ Although promising, these preliminary findings are not wholly persuasive. Questions remain about proper dosing, frequency, supplement formulation, and duration of treatment. Larger comparative trials are needed to more conclusively determine whether turmeric effectively reduces pain and improves functional outcomes in patients with knee $\mathrm{OA}$. A definitive recommendation at this time is not possible based on the available evidence. 


\section{Conclusion}

A review of current literature makes clear the extensive and varied approaches different providers have taken to the problem of knee OA. Perhaps because of its high prevalence and the multitude of providers treating the disease, there are no uniform guidelines for appropriate management suitable across all clinical scenarios. Even within the category of noninterventional management, there exists a diverse array of treatment options. However, as outlined in this review, certain prescriptions bear more consideration than others. Resistance strength training, formal walking programs, and weight loss each have a strong body of evidence supporting their effectiveness at reducing pain and improving physical function in patients with knee OA. Manual mobilization, acupuncture, and knee orthotic devices also appear to be effective in some number of patients, although a more robust set of comparative trials would be helpful in clarifying the true extent of their benefits. Medications represent another important category of management. As discussed above, topicals are often preferable to oral agents due to potential organ toxicity with many of the drugs typically used to treat chronic musculoskeletal pain. Nutritional supplements, therapeutic ultrasound, and electrical stimulation are less strongly supported, although certainly these have proven marginally effective in a subset of patients as well. Despite the breadth of available treatments, a safe and universally effective protocol remains elusive. In its absence, the singular focus of clinical providers must be on improving pain and reducing disability through individualized attention to the patient and rigorous review of the scientific evidence as it continues to emerge.

\section{Conflict of Interest}

None.

\section{References}

1 Dillon CF, Rasch EK, Gu Q, Hirsch R. Prevalence of knee osteoarthritis in the United States: arthritis data from the Third National Health and Nutrition Examination Survey 1991-94. J Rheumatol 2006;33(11):2271-2279

2 Lawrence RC, Felson DT, Helmick CG, et al; National Arthritis Data Workgroup. Estimates of the prevalence of arthritis and other rheumatic conditions in the United States. Part II. Arthritis Rheum 2008;58(01):26-35

3 Felson DT, Naimark A, Anderson J, Kazis L, Castelli W, Meenan RF. The prevalence of knee osteoarthritis in the elderly. The Framingham Osteoarthritis Study. Arthritis Rheum 1987;30(08):914-918

4 Felson DT, Lawrence RC, Dieppe PA, et al. Osteoarthritis: new insights. Part 1: the disease and its risk factors. Ann Intern Med 2000;133(08):635-646

5 Feng JE, Novikov D, Anoushiravani AA, Schwarzkopf R. Total knee arthroplasty: improving outcomes with a multidisciplinary approach. J Multidiscip Healthc 2018;11:63-73

6 Zhang W, Nuki G, Moskowitz RW, et al. OARSI recommendations for the management of hip and knee osteoarthritis: part III: Changes in evidence following systematic cumulative update of research published through January 2009. Osteoarthritis Cartilage 2010;18(04):476-499

7 Lespasio MJ, Piuzzi NS, Husni ME, Muschler GF, Guarino A, Mont MA. Knee osteoarthritis: a primer. Perm J 2017;21:21
8 Sosa-Reina MD, Nunez-Nagy S, Gallego-IzquierdoT, Pecos-Martín D, Monserrat J, Álvarez-Mon M. Effectiveness of therapeutic exercise in fibromyalgia syndrome: a systematic review and meta-analysis of randomized clinical trials. BioMed Res Int 2017;2017:2356346

9 Saragiotto BT, Maher CG, Yamato TP, et al. Motor control exercise for chronic non-specific low-back pain. Cochrane Database Syst Rev 2016;2016(01):CD012004

10 Naugle KM, Fillingim RB, Riley JL III. A meta-analytic review of the hypoalgesic effects of exercise. J Pain 2012;13(12):1139-1150

11 Deyle GD, Allison SC, Matekel RL, et al. Physical therapy treatment effectiveness for osteoarthritis of the knee: a randomized comparison of supervised clinical exercise and manual therapy procedures versus a home exercise program. Phys Ther 2005;85 (12):1301-1317

12 Jan MH, Lin JJ, Liau JJ, Lin YF, Lin DH. Investigation of clinical effects of high- and low-resistance training for patients with knee osteoarthritis: a randomized controlled trial. Phys Ther 2008;88(04):427-436

13 Latham N, Liu C-J. Strength training in older adults: the benefits for osteoarthritis. Clin Geriatr Med 2010;26(03):445-459

14 Newberry SJFJ, SooHoo NF, Booth M, Marks J, Motala A, Apaydin E, Chen C, Raaen L, Shanman R, Shekelle PG. Treatment of osteoarthritis of the knee: an updated review. AHRQ Comparative Effectiveness Reviews. 2017; Report No.: 17-EHCO11-EF

15 Fransen M, McConnell S, Harmer AR, Van der Esch M, Simic M, Bennell KL. Exercise for osteoarthritis of the knee: a Cochrane systematic review. Br J Sports Med 2015;49(24):1554-1557

16 Physical activity. Fact sheet. World Health Organization. 2018

17 Escalante Y, García-Hermoso A, Saavedra JM. Effects of exercise on functional aerobic capacity in lower limb osteoarthritis: a systematic review. J Sci Med Sport 2011;14(03):190-198

18 Roddy E, Zhang W, Doherty M. Aerobic walking or strengthening exercise for osteoarthritis of the knee? A systematic review. Ann Rheum Dis 2005;64(04):544-548

19 Talbot LA, Gaines JM, Huynh TN, Metter EJ. A home-based pedometer-driven walking program to increase physical activity in older adults with osteoarthritis of the knee: a preliminary study. J Am Geriatr Soc 2003;51(03):387-392

20 Kovar PA, Allegrante JP, MacKenzie CR, Peterson MGE, Gutin B, Charlson ME. Supervised fitness walking in patients with osteoarthritis of the knee. A randomized, controlled trial. Ann Intern Med 1992;116(07):529-534

21 van Baar ME, Dekker J, Oostendorp RAB, Bijl D, Voorn TB, Bijlsma JWJ. Effectiveness of exercise in patients with osteoarthritis of hip or knee: nine months' follow up. Ann Rheum Dis 2001;60 (12):1123-1130

22 Sullivan T, Allegrante JP, Peterson MG, Kovar PA, MacKenzie CR. Oneyear followup of patients with osteoarthritis of the knee who participated in a program of supervised fitness walking and supportive patient education. Arthritis Care Res 1998;11(04):228-233

23 Hurley MV, Walsh NE, Mitchell HL, et al. Clinical effectiveness of a rehabilitation program integrating exercise, self-management, and active coping strategies for chronic knee pain: a cluster randomized trial. Arthritis Rheum 2007;57(07):1211-1219

24 Regnaux JP, Lefevre-Colau MM, Trinquart L, et al. High-intensity versus low-intensity physical activity or exercise in people with hip or knee osteoarthritis. Cochrane Database Syst Rev 2015; 2015(10):CD010203

25 Wyatt FB, Milam S, Manske RC, Deere R. The effects of aquatic and traditional exercise programs on persons with knee osteoarthritis. J Strength Cond Res 2001;15(03):337-340

26 Silva LE, Valim V, Pessanha AP, et al. Hydrotherapy versus conventional land-based exercise for the management of patients with osteoarthritis of the knee: a randomized clinical trial. Phys Ther 2008;88(01):12-21

27 Foley A, Halbert J, Hewitt T, Crotty M. Does hydrotherapy improve strength and physical function in patients with osteoarthritis-a randomised controlled trial comparing a gym based 
and a hydrotherapy based strengthening programme. Ann Rheum Dis 2003;62(12):1162-1167

28 Cadmus L, Patrick MB, Maciejewski ML, Topolski T, Belza B, Patrick DL. Community-based aquatic exercise and quality of life in persons with osteoarthritis. Med Sci Sports Exerc 2010;42(01):8-15

29 Lu M, Su Y, Zhang Y, et al. Effectiveness of aquatic exercise for treatment of knee osteoarthritis: systematic review and metaanalysis. Z Rheumatol 2015;74(06):543-552

30 Lange AK, Vanwanseele B, Fiatarone Singh MA. Strength training for treatment of osteoarthritis of the knee: a systematic review. Arthritis Rheum 2008;59(10):1488-1494

31 Alnahdi AH, Zeni JA, Snyder-Mackler L. Muscle impairments in patients with knee osteoarthritis. Sports Health 2012;4(04): 284-292

32 Baker KR, Nelson ME, Felson DT, Layne JE, Sarno R, Roubenoff R. The efficacy of home based progressive strength training in older adults with knee osteoarthritis: a randomized controlled trial. J Rheumatol 2001;28(07):1655-1665

33 O'Reilly SC, Muir KR, Doherty M. Effectiveness of home exercise on pain and disability from osteoarthritis of the knee: a randomised controlled trial. Ann Rheum Dis 1999;58(01):15-19

34 Skoffer B, Dalgas U, Mechlenburg I. Progressive resistance training before and after total hip and knee arthroplasty: a systematic review. Clin Rehabil 2015;29(01):14-29

35 Swank AM, Kachelman JB, Bibeau W, et al. Prehabilitation before total knee arthroplasty increases strength and function in older adults with severe osteoarthritis. J Strength Cond Res 2011;25 (02):318-325

36 Apold H, Meyer HE, Nordsletten L, Furnes O, Baste V, Flugsrud GB. Weight gain and the risk of knee replacement due to primary osteoarthritis: a population based, prospective cohort study of 225,908 individuals. Osteoarthritis Cartilage 2014;22(05):652-658

37 Riddle DL, Stratford PW. Body weight changes and corresponding changes in pain and function in persons with symptomatic knee osteoarthritis: a cohort study. Arthritis Care Res (Hoboken) 2013;65(01):15-22

38 Jiang L, Tian W, Wang Y, et al. Body mass index and susceptibility to knee osteoarthritis: a systematic review and meta-analysis. Joint Bone Spine 2012;79(03):291-297

39 Wluka AE, Lombard CB, Cicuttini FM. Tackling obesity in knee osteoarthritis. Nat Rev Rheumatol 2013;9(04):225-235

40 Miller GD, Nicklas BJ, Davis C, Loeser RF, Lenchik L, Messier SP. Intensive weight loss program improves physical function in older obese adults with knee osteoarthritis. Obesity (Silver Spring) 2006;14(07):1219-1230

41 Christensen R, Henriksen M, Leeds AR, et al. Effect of weight maintenance on symptoms of knee osteoarthritis in obese patients: a twelve-month randomized controlled trial. Arthritis Care Res (Hoboken) 2015;67(05):640-650

42 Christensen R, Bartels EM, Astrup A, Bliddal H. Effect of weight reduction in obese patients diagnosed with knee osteoarthritis: a systematic review and meta-analysis. Ann Rheum Dis 2007;66 (04):433-439

43 Foy CG, Lewis CE, Hairston KG, et al; Look AHEAD Research Group. Intensive lifestyle intervention improves physical function among obese adults with knee pain: findings from the Look AHEAD trial. Obesity (Silver Spring) 2011;19(01):83-93

44 Messier SP, Mihalko SL, Legault C, et al. Effects of intensive diet and exercise on knee joint loads, inflammation, and clinical outcomes among overweight and obese adults with knee osteoarthritis: the IDEA randomized clinical trial. JAMA 2013; 310(12):1263-1273

45 Clegg DO, Reda DJ, Harris CL, et al. Glucosamine, chondroitin sulfate, and the two in combination for painful knee osteoarthritis. N Engl J Med 2006;354(08):795-808

46 Jafarzadeh SR, Clancy M, Li JS, et al. Changes in the structural features of osteoarthritis in a year of weight loss. Osteoarthritis Cartilage 2018;26(06):775-782
47 Gersing AS, Solka M, Joseph GB, et al. Progression of cartilage degeneration and clinical symptoms in obese and overweight individuals is dependent on the amount of weight loss: 48-month data from the Osteoarthritis Initiative. Osteoarthritis Cartilage 2016;24(07):1126-1134

48 Gudbergsen H, Boesen M, Lohmander LS, et al. Weight loss is effective for symptomatic relief in obese subjects with knee osteoarthritis independently of joint damage severity assessed by high-field MRI and radiography. Osteoarthritis Cartilage 2012;20(06):495-502

49 NHLBI Obesity Education Initiative Expert Panel on the Identification, Evaluation, and Treatment of Obesity in Adults (US). Clinical Guidelines on the Identification, Evaluation, and Treatment of Overweight and Obesity in Adults: The Evidence Report. Bethesda (MD): National Heart, Lung, and Blood Institute 1998 Sep.

50 Messier SP, Loeser RF, Miller GD, et al. Exercise and dietary weight loss in overweight and obese older adults with knee osteoarthritis: the Arthritis, Diet, and Activity Promotion Trial. Arthritis Rheum 2004;50(05):1501-1510

51 McAlindon TE, Bannuru RR, Sullivan MC, et al. OARSI guidelines for the non-surgical management of knee osteoarthritis. Osteoarthritis Cartilage 2014;22(03):363-388

52 Raja K, Dewan N. Efficacy of knee braces and foot orthoses in conservative management of knee osteoarthritis a systematic review. Am J Phys Med Rehabil 2011;90(03):247-262

53 Duivenvoorden T, Brouwer RW, van Raaij TM, Verhagen AP, Verhaar JA, Bierma-Zeinstra SM. Braces and orthoses for treating osteoarthritis of the knee. Cochrane Database Syst Rev 2015; 2015(03):CD004020

54 Komistek RD, Dennis DA, Northcut EJ, Wood A, Parker AW, Traina $\mathrm{SM}$. An in vivo analysis of the effectiveness of the osteoarthritic knee brace during heel-strike of gait.J Arthroplasty 1999;14(06):738-742

55 Birmingham TB, Kramer JF, Kirkley A, Inglis JT, Spaulding SJ, Vandervoort AA. Knee bracing for medial compartment osteoarthritis: effects on proprioception and postural control. Rheumatology (Oxford) 2001;40(03):285-289

56 Draper ERC, Cable JM, Sanchez-Ballester J, Hunt N, Robinson JR, Strachan RK. Improvement in function after valgus bracing of the knee. An analysis of gait symmetry. J Bone Joint Surg Br 2000;82 (07):1001-1005

57 Bennell KL, Bowles K-A, Payne C, et al. Lateral wedge insoles for medial knee osteoarthritis: 12 month randomised controlled trial. BMJ 2011;342(7808):d2912

58 Pham T, Maillefert JF, Hudry C, et al. Laterally elevated wedged insoles in the treatment of medial knee osteoarthritis. A twoyear prospective randomized controlled study. Osteoarthritis Cartilage 2004;12(01):46-55

59 Malvankar S, Khan WS, Mahapatra A, Dowd GS. How effective are lateral wedge orthotics in treating medial compartment osteoarthritis of the knee? A systematic review of the recent literature. Open Orthop J 2012;6:544-547

60 Erhart JC, Mündermann A, Elspas B, Giori NJ, Andriacchi TP. Changes in knee adduction moment, pain, and functionality with a variable-stiffness walking shoe after 6 months. J Orthop Res 2010;28(07):873-879

61 Erhart-Hledik JC, Elspas B, Giori NJ, Andriacchi TP. Effect of variable-stiffness walking shoes on knee adduction moment, pain, and function in subjects with medial compartment knee osteoarthritis after 1 year. J Orthop Res 2012;30(04):514-521

62 Radzimski AO, Mündermann A, Sole G. Effect of footwear on the external knee adduction moment - a systematic review. Knee 2012;19(03):163-175

63 Manheimer E, Cheng K, Linde K, et al. Acupuncture for peripheral joint osteoarthritis. Cochrane Database Syst Rev 2010;(01): CD001977

64 Selfe TK, Taylor AG. Acupuncture and osteoarthritis of the knee: a review of randomized, controlled trials. Fam Community Health 2008;31(03):247-254 
65 Cao L, Zhang X-L, Gao Y-S, Jiang Y. Needle acupuncture for osteoarthritis of the knee. A systematic review and updated meta-analysis. Saudi Med J 2012;33(05):526-532

66 Ulus Y, Tander B, Akyol Y, et al. Therapeutic ultrasound versus sham ultrasound for the management of patients with knee osteoarthritis: a randomized double-blind controlled clinical study. Int J Rheum Dis 2012;15(02):197-206

67 Loyola-Sánchez A, Richardson J, MacIntyre NJ. Efficacy of ultrasound therapy for the management of knee osteoarthritis: a systematic review with meta-analysis. Osteoarthritis Cartilage 2010;18(09):1117-1126

68 Rutjes AW, Nüesch E, Sterchi R, Jüni P. Therapeutic ultrasound for osteoarthritis of the knee or hip. Cochrane Database Syst Rev 2010;(01):CD003132

69 Giggins O, Fullen B, Coughlan G. Neuromuscular electrical stimulation in the treatment of knee osteoarthritis: a systematic review and meta-analysis. Clin Rehabil 2012;26(10):867-881

70 Hochberg MC, Altman RD, April KT, et al; American College of Rheumatology. American College of Rheumatology 2012 recommendations for the use of nonpharmacologic and pharmacologic therapies in osteoarthritis of the hand, hip, and knee. Arthritis Care Res (Hoboken) 2012;64(04):465-474

71 Batavia M. Contraindications for superficial heat and therapeutic ultrasound: do sources agree? Arch Phys Med Rehabil 2004;85 (06):1006-1012

72 Jones I, Johnson MI. Transcutaneous electrical nerve stimulation. Continuing Education in Anesthesia, Critical Care \& Pain 2009; 9(04):130-135

73 Jansen MJ, Viechtbauer W, Lenssen AF, Hendriks EJM, de Bie RA. Strength training alone, exercise therapy alone, and exercise therapy with passive manual mobilisation each reduce pain and disability in people with knee osteoarthritis: a systematic review. J Physiother 2011;57(01):11-20

74 Deyle GD, Henderson NE, Matekel RL, Ryder MG, Garber MB, Allison SC. Effectiveness of manual physical therapy and exercise in osteoarthritis of the knee. A randomized, controlled trial. Ann Intern Med 2000;132(03):173-181

75 Hughes SL, Seymour RB, Campbell RT, et al. Long-term impact of Fit and Strong! on older adults with osteoarthritis. Gerontologist 2006;46(06):801-814

76 Ringdahl E, Pandit S. Treatment of knee osteoarthritis. Am Fam Physician 2011;83(11):1287-1292

77 Peterson K, McDonagh M, Thakurta S, et al. Drug class review: nonsteroidal antiinflammatory drugs (NSAIDs): final update 4 report. Drug Class Reviews 2010

78 van Laar M, Pergolizzi JV Jr, Mellinghoff $\mathrm{H}-\mathrm{U}$, et al. Pain treatment in arthritis-related pain: beyond NSAIDs. Open Rheumatol J 2012;6:320-330

79 Altman RD, Barthel HR. Topical therapies for osteoarthritis. Drugs 2011;71(10):1259-1279

80 Derry S, Conaghan P, Da Silva JAP, Wiffen PJ, Moore RA. Topical NSAIDs for chronic musculoskeletal pain in adults. Cochrane Database Syst Rev 2016;4:CD007400

81 Bookman AA, Williams KS, Shainhouse JZ. Effect of a topical diclofenac solution for relieving symptoms of primary osteoarthritis of the knee: a randomized controlled trial. CMAJ 2004; 171(04):333-338

82 Roth SH, Shainhouse JZ. Efficacy and safety of a topical diclofenac solution (pennsaid) in the treatment of primary osteoarthritis of the knee: a randomized, double-blind, vehicle-controlled clinical trial. Arch Intern Med 2004;164(18):2017-2023

83 Sardana V, Burzynski J, Zalzal P. Safety and efficacy of topical ketoprofen in transfersome gel in knee osteoarthritis: a systematic review. Musculoskelet Care 2017;15(02):114-121

84 Deal CL, Schnitzer TJ, Lipstein E, et al. Treatment of arthritis with topical capsaicin: a double-blind trial. Clin Ther 1991;13(03): 383-395
85 Kosuwon W, Sirichatiwapee W, Wisanuyotin T, Jeeravipoolvarn P, Laupattarakasem W. Efficacy of symptomatic control of knee osteoarthritis with $0.0125 \%$ of capsaicin versus placebo. J Med Assoc Thai 2010;93(10):1188-1195

86 Kivitz A, Fairfax M, Sheldon EA, et al. Comparison of the effectiveness and tolerability of lidocaine patch $5 \%$ versus celecoxib for osteoarthritis-related knee pain: post hoc analysis of a 12 week, prospective, randomized, active-controlled, open-label, parallelgroup trial in adults. Clin Ther 2008;30(12):2366-2377

87 Jordan KM, Arden NK, Doherty M, et al; Standing Committee for International Clinical Studies Including Therapeutic Trials ESCISIT. EULAR Recommendations 2003: an evidence based approach to the management of knee osteoarthritis: Report of a Task Force of the Standing Committee for International Clinical Studies Including Therapeutic Trials (ESCISIT). Ann Rheum Dis 2003;62(12):1145-1155

88 Zhang W, Moskowitz RW, Nuki G, et al. OARSI recommendations for the management of hip and knee osteoarthritis, Part II: OARSI evidence-based, expert consensus guidelines. Osteoarthritis Cartilage 2008;16(02):137-162

89 Goldenberg DL. Pharmacological treatment of fibromyalgia and other chronic musculoskeletal pain. Best Pract Res Clin Rheumatol 2007;21(03):499-511

90 Brown JP, Boulay LJ. Clinical experience with duloxetine in the management of chronic musculoskeletal pain. A focus on osteoarthritis of the knee. Ther Adv Musculoskelet Dis 2013;5(06):291-304

91 Hudson B, Williman JA, Stamp LK, et al. Nortriptyline in knee osteoarthritis (NortIKA Study): study protocol for a randomised controlled trial. Trials 2015;16:448

92 Lyttle JR, Urquhart DM, Cicuttini FM, Wluka AE. Antidepressants for osteoarthritis. Cochrane Database Syst Rev 2016;2016(04):1-13

93 Crofford LJ. Adverse effects of chronic opioid therapy for chronic musculoskeletal pain. Nat Rev Rheumatol 2010;6(04):191-197

94 Cepeda MS, Camargo F, Zea C, Valencia L. Tramadol for osteoarthritis: a systematic review and metaanalysis. J Rheumatol 2007; 34(03):543-555

95 da Costa BR, Nüesch E, Kasteler R, et al. Oral or transdermal opioids for osteoarthritis of the knee or hip. Cochrane Database Syst Rev 2014;(09):CD003115

96 Krebs EE, Gravely A, Nugent S, et al. Effect of opioid vs nonopioid medications on pain-related function in patients with chronic back pain or hip or knee osteoarthritis pain: the SPACE randomized clinical trial. JAMA 2018;319(09):872-882

97 Bruyere O, Reginster J-Y. Glucosamine and chondroitin sulfate as therapeutic agents for knee and hip osteoarthritis. Drugs Aging 2007;24(07):573-580

98 Fransen M, Agaliotis M, Nairn L, et al; LEGS study collaborative group. Glucosamine and chondroitin for knee osteoarthritis: a double-blind randomised placebo-controlled clinical trial evaluating single and combination regimens. Ann Rheum Dis 2015;74(05):851-858

99 Wandel S, Jüni P, Tendal B, et al. Effects of glucosamine, chondroitin, or placebo in patients with osteoarthritis of hip or knee: network meta-analysis. BMJ 2010;341:c4675

100 Henrotin Y, Priem F, Mobasheri A. Curcumin: a new paradigm and therapeutic opportunity for the treatment of osteoarthritis: curcumin for osteoarthritis management. Springerplus 2013;2(01):56

101 Onakpoya IJ, Spencer EA, Perera R, Heneghan CJ. Effectiveness of curcuminoids in the treatment of knee osteoarthritis: a systematic review and meta-analysis of randomized clinical trials. Int J Rheum Dis 2017;20(04):420-433

102 Nakagawa Y, Mukai S, Yamada S, et al. Short-term effects of highlybioavailable curcumin for treating knee osteoarthritis: a randomized, double-blind, placebo-controlled prospective study.J Orthop Sci 2014;19(06):933-939

103 Panahi Y, Rahimnia AR, Sharafi M, Alishiri G, Saburi A, Sahebkar A. Curcuminoid treatment for knee osteoarthritis: a randomized double-blind placebo-controlled trial. Phytother Res 2014;28 (11):1625-1631 